\title{
On strong law of large numbers and growth rate for a class of random variables
}

\author{
Yan Shen, Jie Yang and Shuhe $\mathrm{Hu}^{*}$
}

\section{*Correspondence:} hushuhe@263.net

School of Mathematical Science, Anhui University, Hefei, 230039, P.R. China

\begin{abstract}
In this paper, we study the strong law of large numbers for a class of random variables satisfying the maximal moment inequality with exponent 2 . Our results embrace the Kolmogorov strong law of large numbers and the Marcinkiewicz strong law of large numbers for this class of random variables. In addition, strong growth rate for weighted sums of this class of random variables is presented.
\end{abstract}

MSC: $60 \mathrm{~F} 15$

Keywords: strong law of large numbers; weighted sums; with exponent 2

\section{Introduction}

Let $\left\{X_{n}, n \geq 1\right\}$ be a sequence of random variables defined on a fixed probability space $(\Omega, \mathcal{F}, P) . S_{n}=\sum_{i=1}^{n} X_{i}, n \geq 1, S_{0}=0$. Let $\left\{a_{n}, n \geq 1\right\}$ and $\left\{b_{n}, n \geq 1\right\}$ be sequences of constant with $0<b_{n} \uparrow \infty$. Then $\left\{a_{n} X_{n}, n \geq 1\right\}$ is said to obey the general strong law of large numbers (SLLN) with norming constant $\left\{b_{n}, n \geq 1\right\}$ if the normed weighted sums

$$
\frac{1}{b_{n}} \sum_{i=1}^{n} a_{i}\left(X_{i}-E X_{i}\right) \rightarrow 0 \quad \text { almost surely (a.s.) }
$$

holds. Note that the SLLN of the form (1.1) embraces the Kolmogorov SLLN $\left(b_{n}=n, a_{n}=1\right)$ and the Marcinkiewicz SLLN $\left(b_{n}=n^{1 / r}, a_{n}=1, r>0\right)$. When $b_{n}=\sum_{i=1}^{n} a_{i}$, fundamental results for the SLLN were obtained.

Under an independent assumption, many SLLNs for the weighted sums are obtained. One can refer to Adler and Rosalsky [1], Chow and Teicher [2], Fernholz and Teicher [3], Jamison et al. [4] and Teicher [5].

Under a pairwise independent assumption, Rosalsky [6] obtained some SLLNs for weighted sums of pairwise independent and identically distributed random variables. Sung [7] obtained sufficient conditions for (1.1) if $\left\{X_{n}, n \geq 1\right\}$ is a sequence of pairwise independent random variables satisfying $\int_{0}^{\infty} x^{p-1} \sup _{n \geq 1} P\left(\left|X_{n}\right|>x\right) d x<\infty$. Sung [8] presented the following result: $\frac{1}{a_{n}} \sum_{i=1}^{n}\left(X_{i}-E X_{i} I\left(\left|X_{i}\right| \leq a_{i}\right)\right) \rightarrow 0$ a.s., where $\left\{a_{n}\right\}$ is a sequence of positive constants with $\frac{a_{n}}{n} \uparrow$ and $\left\{X_{n}\right\}$ is a sequence of pairwise independent and identically distributed random variables.

For more details about strong limit theorems for dependent case, one can refer to $\mathrm{Wu}$ [9], Wu and Jiang [10], Hu et al. [11], Shen et al. [12], Zhou et al. [13] and Zhou [14], and so forth.

Recently Sung [15] gave the following definition.

\section{Springer}

(02013 Shen et al.; licensee Springer. This is an Open Access article distributed under the terms of the Creative Commons Attribution License (http://creativecommons.org/licenses/by/2.0), which permits unrestricted use, distribution, and reproduction in any medium, provided the original work is properly cited. 
Definition 1.1 (Sung [15]) A random variable sequence $\left\{X_{n}, n \geq 1\right\}$ is said to satisfy the maximal moment inequality with exponent 2 if for all $n \geq m \geq 1$, there exists a constant $C$ independent of $n$ and $m$ such that

$$
E\left(\max _{m \leq k \leq n}\left|\sum_{i=m}^{k} X_{i}\right|^{2}\right) \leq C \sum_{i=m}^{n} E X_{i}^{2} .
$$

We can see that a wide class of mean zero random variables satisfies (1.2). Inspired by Sung $[7,15]$, we establish SLLN of the form (1.1) for a class of random variables satisfying the maximal moment inequality with exponent 2 .

The rest of the paper is organized as follows. In Section 2, some preliminary definition and lemmas are presented. In Section 3, main results and their proofs are provided.

Throughout the paper, let $I(A)$ be the indicator function of the set $A$. $C$ denotes a positive constant not depending on $n$, which may be different in various places. Let $\left\{a_{n}, n \geq 1\right\}$ and $\left\{b_{n}, n \geq 1\right\}$ be sequences of positive numbers, $a_{n} \ll b_{n}$ represents that there exists a constant $C>0$ such that $a_{n} \leq C b_{n}$ for all $n$.

\section{Preliminaries}

The following lemmas and definition will be needed in this paper.

Lemma 2.1 (Sung [7]) Let $\left\{X_{n}, n \geq 1\right\}$ be a sequence of random variables and put $G(x)=$ $\sup _{n \geq 1} P\left(\left|X_{n}\right|>x\right)$ for $x \geq 0$. Assume that $\int_{0}^{\infty} x^{p-1} G(x) d x<\infty$ for some $1 \leq p<2$. Then

(i) $\sum_{n=1}^{\infty} P\left(\left|X_{n}\right|>n^{1 / p}\right)<\infty$.

(ii) $\sum_{n=1}^{\infty} E X_{n}^{2} I\left(\left|X_{n}\right| \leq n^{1 / p}\right) / n^{2 / p}<\infty$.

(iii) $E X_{n} I\left(\left|X_{n}\right|>c_{n}\right) \rightarrow 0$ for any sequence $\left\{c_{n}, n \geq 1\right\}$ satisfying $c_{n} \rightarrow \infty$.

Lemma 2.2 (Sung [15]) Let $\left\{X_{n}, n \geq 1\right\}$ be a sequence of random variables satisfying the maximal moment inequality with exponent 2 . If $\sum_{n=1}^{\infty} E X_{n}^{2}<\infty$, then $\sum_{n=1}^{\infty} X_{n}$ converges almost surely.

Definition 2.3 A random variable sequence $\left\{X_{n}, n \geq 1\right\}$ is said to be stochastically dominated by a random variable $X$ if there exists a constant $C$ such that

$$
P\left(\left|X_{n}\right|>x\right) \leq C P(|X|>x)
$$

for all $x \geq 0$ and $n \geq 1$.

Lemma 2.4 Let $\left\{X_{n}, n \geq 1\right\}$ be a sequence of random variables which is stochastically dominated by a random variable $X$. For any $\alpha>0$ and $b>0$, the following statement holds:

$$
\begin{aligned}
& E\left|X_{n}\right|^{\alpha} I\left(\left|X_{n}\right| \leq b\right) \leq C\left\{E|X|^{\alpha} I(|X| \leq b)+b^{\alpha} P(|X|>b)\right\}, \\
& E\left|X_{n}\right|^{\alpha} I\left(\left|X_{n}\right|>b\right) \leq C E|X|^{\alpha} I(|X|>b),
\end{aligned}
$$

where $C$ is a positive constant. 
Lemma $2.5(\mathrm{Hu}[16])$ Let $b_{1}, b_{2}, \ldots$ be a nondecreasing unbounded sequence of positive numbers. Let $\alpha_{1}, \alpha_{2}, \ldots$ be nonnegative numbers, and $\Lambda_{k}=\alpha_{1}+\cdots+\alpha_{k}$ for $k \geq 1$. Let $r$ be a fixed positive number. Assume that for each $n \geq 1$,

$$
E\left(\max _{1 \leq k \leq n}\left|S_{k}\right|\right)^{r} \leq C \sum_{k=1}^{n} \alpha_{k}
$$

If

$$
\begin{aligned}
& \sum_{l=1}^{\infty} \Lambda_{l}\left(\frac{1}{b_{l}^{r}}-\frac{1}{b_{l+1}^{r}}\right)<\infty, \\
& \frac{\Lambda_{n}}{b_{n}^{r}} \text { is bounded, }
\end{aligned}
$$

then

$$
\lim _{n \rightarrow \infty} \frac{S_{n}}{b_{n}}=0 \quad \text { a.s. }
$$

and with the growth rate

$$
\frac{S_{n}}{b_{n}}=O\left(\frac{\beta_{n}}{b_{n}}\right) \quad \text { a.s. }
$$

where

$$
\beta_{n}=\max _{1 \leq k \leq n} b_{k} v_{k}^{\delta / r}, \quad \forall 0<\delta<1, v_{n}=\sum_{k=n}^{\infty} \frac{\alpha_{k}}{b_{k}^{r}}, \lim _{n \rightarrow \infty} \frac{\beta_{n}}{b_{n}}=0 .
$$

And

$$
\begin{aligned}
& E\left(\max _{1 \leq l \leq n}\left|\frac{S_{l}}{b_{l}}\right|\right)^{r} \leq 4 C \sum_{l=1}^{n} \frac{\alpha_{l}}{b_{l}^{r}}<\infty, \\
& E\left(\sup _{l \geq 1}\left|\frac{S_{l}}{b_{l}}\right|\right)^{r} \leq 4 C \sum_{l=1}^{\infty} \frac{\alpha_{l}}{b_{l}^{r}}<\infty .
\end{aligned}
$$

If we further assume that $\alpha_{n}>0$ for infinitely many $n$, then

$$
E\left(\sup _{l \geq 1}\left|\frac{S_{l}}{\beta_{l}}\right|\right)^{r} \leq 4 C \sum_{l=1}^{\infty} \frac{\alpha_{l}}{\beta_{l}^{r}}<\infty .
$$

Proof It follows from Corollary 2.1.1 of $\mathrm{Hu}$ [16] that (2.6)-(2.8) hold. By (2.3) and Theorem 1.1 of Fazekas and Klesov [17], we have

$$
E\left(\max _{1 \leq l \leq n}\left|\frac{S_{l}}{b_{l}}\right|\right)^{r} \leq 4 C \sum_{l=1}^{n} \frac{\alpha_{l}}{b_{l}^{r}} \leq 4 C \sum_{l=1}^{\infty} \frac{\alpha_{l}}{b_{l}^{r}}<\infty .
$$


Therefore

$$
E\left(\sup _{l \geq 1}\left|\frac{S_{l}}{b_{l}}\right|\right)^{r}=\lim _{n \rightarrow \infty} E\left(\max _{1 \leq l \leq n}\left|\frac{S_{l}}{b_{l}}\right|\right)^{r} \leq 4 C \sum_{l=1}^{\infty} \frac{\alpha_{l}}{b_{l}^{r}}<\infty
$$

following from the monotone convergence theorem of Rao [18]. Equation (2.11) follows from the proof of Lemma 1.2 of $\mathrm{Hu}$ and $\mathrm{Hu}[19]$.

\section{Main results}

Theorem 3.1 Let $\left\{X_{n}, n \geq 1\right\}$ be a sequence of random variables and put $G(x)=$ $\sup _{n \geq 1} P\left(\left|X_{n}\right|>x\right)$ for $x>0$. Denote $Y_{n}=-n^{1 / p} I\left(X_{n}<-n^{1 / p}\right)+X_{n} I\left(\left|X_{n}\right| \leq n^{1 / p}\right)+n^{1 / p} I\left(X_{n}>\right.$ $\left.n^{\frac{1}{p}}\right), n \geq 1$, where $p$ is a positive constant. Let $\left\{a_{n}, n \geq 1\right\}$ and $\left\{b_{n}, n \geq 1\right\}$ be sequences of positive numbers with $b_{n} \uparrow \infty$. Suppose that $\left\{\frac{a_{n}}{b_{n}}\left(Y_{n}-E Y_{n}\right), n \geq 1\right\}$ satisfies the maximal moment inequality with exponent 2 . Assume that the following two conditions hold:

$$
\begin{aligned}
& \sum_{i=1}^{n} a_{i}=O\left(b_{n}\right), \\
& \frac{a_{n}}{b_{n}}=O\left(n^{-1 / p}\right) \quad \text { for some } 1 \leq p<2 . \\
& \text { If } \int_{0}^{\infty} x^{p-1} G(x) d x<\infty, \text { then } \\
& \lim _{n \rightarrow \infty} \frac{1}{b_{n}} \sum_{i=1}^{n} a_{i}\left(X_{i}-E X_{i}\right)=0 \quad \text { a.s. }
\end{aligned}
$$

Proof By Lemma 2.1(i),

$$
\sum_{n=1}^{\infty} P\left(X_{n} \neq Y_{n}\right)=\sum_{n=1}^{\infty} P\left(\left|X_{n}\right|>n^{1 / p}\right)<\infty .
$$

Therefore $P\left(X_{n} \neq Y_{n}\right.$, i.o. $)=0$ follows from the Borel-Cantelli lemma and (3.4). Thus (3.3) is equivalent to the following:

$$
\lim _{n \rightarrow \infty} \frac{1}{b_{n}} \sum_{i=1}^{n} a_{i}\left(Y_{i}-E X_{i}\right)=0 \quad \text { a.s. }
$$

So, in order to prove (3.3), we need only to prove

$$
\lim _{n \rightarrow \infty} \frac{1}{b_{n}} \sum_{i=1}^{n} a_{i}\left(Y_{i}-E Y_{i}\right)=0 \quad \text { a.s. }
$$

and

$$
\lim _{n \rightarrow \infty} \frac{1}{b_{n}} \sum_{i=1}^{n} a_{i}\left(E X_{i}-E Y_{i}\right)=0 .
$$


Firstly, we prove (3.6). In view of Lemma 2.1(i), (ii) and (3.2), we have

$$
\begin{aligned}
\sum_{n=1}^{\infty} E\left(\frac{a_{n}}{b_{n}}\left(Y_{n}-E Y_{n}\right)\right)^{2} & \leq \sum_{n=1}^{\infty} \frac{a_{n}^{2}}{b_{n}^{2}} E Y_{n}^{2} \\
& =\sum_{n=1}^{\infty} \frac{a_{n}^{2}}{b_{n}^{2}}\left[E X_{n}^{2} I\left(\left|X_{n}\right| \leq n^{1 / p}\right)+n^{2 / p} E I\left(\left|X_{n}\right|>n^{1 / p}\right)\right] \\
& \ll \sum_{n=1}^{\infty}\left[n^{-2 / p} E X_{n}^{2} I\left(\left|X_{n}\right| \leq n^{1 / p}\right)+P\left(\left|X_{n}\right|>n^{1 / p}\right)\right] \\
& <\infty .
\end{aligned}
$$

Thus it follows by Lemma 2.2 that

$$
\sum_{n=1}^{\infty} \frac{a_{n}}{b_{n}}\left(Y_{n}-E Y_{n}\right) \quad \text { converges a.s. }
$$

By Kronecker's lemma, we can obtain (3.6) immediately.

Secondly, we prove (3.7). By (3.1) and Lemma 2.1(iii), we can get

$$
\lim _{n \rightarrow \infty} \frac{1}{b_{n}} \sum_{i=1}^{n} a_{i} E X_{i} I\left(\left|X_{i}\right|>i^{1 / p}\right)=0
$$

By (3.2) and Lemma 2.1(i), we have

$$
\sum_{n=1}^{\infty} \frac{a_{n}}{b_{n}} n^{1 / p} P\left(X_{n}>n^{1 / p}\right) \ll \sum_{n=1}^{\infty} P\left(X_{n}>n^{1 / p}\right)<\sum_{n=1}^{\infty} P\left(\left|X_{n}\right|>n^{1 / p}\right)<\infty
$$

and

$$
\sum_{n=1}^{\infty} \frac{a_{n}}{b_{n}} n^{1 / p} P\left(X_{n}<-n^{1 / p}\right)<\infty
$$

Thus it follows by Kronecker's lemma that

$$
\lim _{n \rightarrow \infty} \frac{1}{b_{n}} \sum_{i=1}^{n} a_{i} i^{1 / p} P\left(X_{i}>i^{1 / p}\right)=0
$$

and

$$
\lim _{n \rightarrow \infty} \frac{1}{b_{n}} \sum_{i=1}^{n} a_{i} i^{1 / p} P\left(X_{i}<-i^{1 / p}\right)=0
$$

Therefore,

$$
\begin{aligned}
\lim _{n \rightarrow \infty} \frac{1}{b_{n}} \sum_{i=1}^{n} a_{i}\left(E X_{i}-E Y_{i}\right) \\
=\lim _{n \rightarrow \infty}\left[\frac{1}{b_{n}} \sum_{i=1}^{n} a_{i} E X_{i} I\left(\left|X_{i}\right|>i^{1 / p}\right)\right.
\end{aligned}
$$




$$
\begin{aligned}
& \left.+\frac{1}{b_{n}} \sum_{i=1}^{n} a_{i} i^{1 / p} P\left(X_{i}<-i^{1 / p}\right)-\frac{1}{b_{n}} \sum_{i=1}^{n} a_{i} i^{1 / p} P\left(X_{i}>i^{1 / p}\right)\right] \\
= & 0
\end{aligned}
$$

follows from (3.9)-(3.11). Hence the result is proved.

Theorem 3.2 Let $\left\{X_{n}, n \geq 1\right\}$ be a sequence of mean zero random variables, which is stochastically dominated by a random variable $X$. Let $\left\{a_{n}, n \geq 1\right\}$ and $\left\{b_{n}, n \geq 1\right\}$ be sequences of positive numbers with $b_{n} \uparrow \infty$. Put $c_{n}=\frac{b_{n}}{a_{n}}$ for $n \geq 1,1<r<2$. Denote $Y_{n}=$ $-c_{n} I\left(X_{n}<-c_{n}\right)+X_{n} I\left(\left|X_{n}\right| \leq c_{n}\right)+c_{n} I\left(X_{n}>c_{n}\right), n \geq 1$, and suppose that $\left\{\frac{a_{n}}{b_{n}}\left(Y_{n}-E Y_{n}\right), n \geq 1\right\}$ satisfies the maximal moment inequality with exponent 2 . Assume that the following two conditions hold:

$$
\begin{aligned}
& E|X|^{r}<\infty, \\
& N(n)=\operatorname{Card}\left\{i: c_{i} \leq n\right\} \ll n^{r}, \quad n \geq 1 .
\end{aligned}
$$

Then

$$
\lim _{n \rightarrow \infty} \frac{1}{b_{n}} \sum_{i=1}^{n} a_{i} X_{i}=0 \quad \text { a.s. }
$$

Proof Let $N(0)=0$. By (3.13), we can see that $c_{n} \rightarrow \infty$ as $n \rightarrow \infty$. By (3.12) and (3.13),

$$
\begin{aligned}
\sum_{n=1}^{\infty} P\left(X_{n} \neq Y_{n}\right) & =\sum_{n=1}^{\infty} P\left(\left|X_{n}\right|>c_{n}\right) \ll \sum_{n=1}^{\infty} P\left(|X|>c_{n}\right) \\
& =\sum_{n=1}^{\infty} \sum_{c_{n} \leq j<c_{n}+1} P\left(|X|>c_{n}\right) \\
& \leq \sum_{j=1}^{\infty} \sum_{j-1<c_{n} \leq j} P(|X|>j-1) \\
& =\sum_{j=1}^{\infty}(N(j)-N(j-1)) \sum_{k=j}^{\infty} P(k-1<|X| \leq k) \\
& =\sum_{k=1}^{\infty} P(k-1<|X| \leq k) \sum_{j=1}^{k}(N(j)-N(j-1)) \\
& =\sum_{k=1}^{\infty} N(k) P(k-1<|X| \leq k) \\
& \ll \sum_{k=1}^{\infty} k^{r} P(k-1<|X| \leq k) \\
& \ll E|X|^{r}<\infty,
\end{aligned}
$$


which implies $P\left(X_{i} \neq Y_{i}\right.$, i.o. $)=0$ from the Borel-Cantelli lemma. So, in order to prove (3.14), we need only to prove

$$
\lim _{n \rightarrow \infty} \frac{1}{b_{n}} \sum_{i=1}^{n} a_{i} Y_{i}=0 \quad \text { a.s. }
$$

By (3.12), (3.13), Lemma 2.4, and the proof of (3.15), we have

$$
\begin{aligned}
\sum_{n=1}^{\infty} & E\left(\frac{a_{n}}{b_{n}}\left(Y_{n}-E Y_{n}\right)\right)^{2} \\
& \leq \sum_{n=1}^{\infty} c_{n}^{-2} E Y_{n}^{2} \\
& =\sum_{n=1}^{\infty} c_{n}^{-2}\left[E X_{n}^{2} I\left(\left|X_{n}\right| \leq c_{n}\right)+E\left(c_{n}^{2} I\left(\left|X_{n}\right|>c_{n}\right)\right)\right] \\
& \ll \sum_{n=1}^{\infty} c_{n}^{-2} E X^{2} I\left(|X| \leq c_{n}\right)+\sum_{n=1}^{\infty} P\left(|X|>c_{n}\right) \\
= & \sum_{n=1}^{\infty} \sum_{c_{n} \leq j<c_{n}+1} c_{n}^{-2} E X^{2} I\left(|X| \leq c_{n}\right)+\sum_{n=1}^{\infty} P\left(|X|>c_{n}\right) \\
\leq & \sum_{j=1}^{\infty} \sum_{j-1<c_{n} \leq j} c_{n}^{-2} E X^{2} I(|X| \leq j)+C \\
& \ll \sum_{j=2}^{\infty}(N(j)-N(j-1))(j-1)^{-2} \sum_{k=1}^{j} E X^{2} I(k-1<|X| \leq k)+C \\
& +\sum_{k=2}^{\infty} E X^{2} I(k-1<|X| \leq k) \sum_{j=k}^{\infty} N(j)\left((j-1)^{-2}-j^{-2}\right)+C \\
& \ll \\
\quad & \sum_{j=2}^{\infty}(N(j)-N(j-1))(j-1)^{-2}\left[E X^{2} I(0<|X| \leq 1)+\sum_{k=2}^{\infty} E X^{2} I(k-1<|X| \leq k)\right]+C \\
& +\sum_{k=2}^{\infty} k^{r-3}+\sum_{k=2}^{\infty} E\left(|X|^{r} k^{2-r} I(k-1<|X| \leq k)\right)+C \\
= & \sum_{j=2}^{\infty}(N(j)-N(j-1))(j-1)^{-2} E X^{2} I(0<|X| \leq 1) \\
& \sum^{2} I(k-1<|X| \leq k) \sum_{j=k}^{\infty}(N(j)-N(j-1))(j-1)^{-2}+C
\end{aligned}
$$


Combining Lemma 2.2, (3.17) and Kronecker's lemma, we can get

$$
\lim _{n \rightarrow \infty} \frac{1}{b_{n}} \sum_{i=1}^{n} a_{i}\left(Y_{i}-E Y_{i}\right)=0 \quad \text { a.s. }
$$

To complete the proof of (3.16), it suffices to show that

$$
\lim _{n \rightarrow \infty} \frac{1}{b_{n}} \sum_{i=1}^{n} a_{i} E Y_{i}=0 .
$$

By (3.12), (3.13) and $E X_{n}=0$, it follows that

$$
\begin{aligned}
\sum_{n=1}^{\infty}\left|\frac{a_{n}}{b_{n}} E Y_{n}\right| & \leq \sum_{n=1}^{\infty} c_{n}^{-1}\left[E\left|X_{n}\right| I\left(\left|X_{n}\right|>c_{n}\right)+E\left(c_{n} I\left(\left|X_{n}\right|>c_{n}\right)\right)\right] \\
& =\sum_{n=1}^{\infty} c_{n}^{-1} E\left|X_{n}\right| I\left(\left|X_{n}\right|>c_{n}\right)+\sum_{n=1}^{\infty} P\left(\left|X_{n}\right|>c_{n}\right) .
\end{aligned}
$$

Observe that

$$
\begin{aligned}
\sum_{n=1}^{\infty} & c_{n}^{-1} E\left|X_{n}\right| I\left(\left|X_{n}\right|>c_{n}\right) \\
\leq & C \sum_{n=1}^{\infty} c_{n}^{-1} E|X| I\left(|X|>c_{n}\right) \\
= & C \sum_{n=1}^{\infty} \sum_{c_{n} \leq j<c_{n}+1} c_{n}^{-1} E|X| I\left(|X|>c_{n}\right) \\
\leq & C \sum_{j=1}^{\infty} \sum_{j-1<c_{n} \leq j} c_{n}^{-1} E|X| I(|X|>j-1) \\
\leq & C \sum_{j=2}^{\infty}(N(j)-N(j-1))(j-1)^{-1} \sum_{n=j-1}^{\infty} E|X| I(n<|X| \leq n+1) \\
\leq & C \sum_{n=1}^{\infty} E|X| I(n<|X| \leq n+1) \sum_{j=2}^{n+1}(N(j)-N(j-1))(j-1)^{-1} \\
\leq & C \sum_{n=1}^{\infty} E|X| I(n<|X| \leq n+1) \sum_{j=2}^{n} j^{r-2}+C \sum_{n=1}^{\infty} n^{r-1} E|X| I(n<|X| \leq n+1) \\
\leq & C \sum_{n=1}^{\infty} E|X| I(n<|X| \leq n+1) \sum_{j=2}^{n} N(j)\left((j-1)^{-1}-j^{-1}\right) \\
& +C \sum_{n=1}^{\infty} E|X| I(n<|X| \leq n+1) \frac{N(n+1)}{n} E|X| I(n<|X| \leq n+1)
\end{aligned}
$$




$$
\begin{aligned}
& \leq C \sum_{n=1}^{\infty} E|X|^{r} I(n<|X| \leq n+1) \\
& \leq C E|X|^{r}<\infty .
\end{aligned}
$$

So, we can get

$$
\sum_{n=1}^{\infty}\left|\frac{a_{n}}{b_{n}} E Y_{n}\right|<\infty
$$

from (3.15), (3.20) and (3.21). Consequently,

$$
\sum_{n=1}^{\infty} \frac{a_{n}}{b_{n}} E Y_{n} \quad \text { converges, }
$$

which implies (3.19) from Kronecker's lemma. We complete the proof of theorem.

Theorem 3.3 Let $\left\{X_{n}, n \geq 1\right\}$ be a sequence of mean zero random variables satisfying the maximal moment inequality with exponent 2 . Denote $Q_{n}=\max _{1 \leq k \leq n} E X_{k}^{2}, n \geq 1$ and $Q_{0}=0$. For $1 \leq p<2$, assume that

$$
\sum_{n=1}^{\infty} \frac{Q_{n}}{n^{2 / p}}<\infty
$$

Then

$$
\lim _{n \rightarrow \infty} \frac{S_{n}}{n^{1 / p}}=0 \quad \text { a.s. }
$$

and with the growth rate

$$
\frac{S_{n}}{n^{1 / p}}=O\left(\frac{\beta_{n}}{n^{1 / p}}\right) \quad \text { a.s. }
$$

where

$$
\begin{aligned}
& \beta_{n}=\max _{1 \leq k \leq n} k^{1 / p} v_{k}^{\delta / 2}, \quad \forall 0<\delta<1, v_{n}=\sum_{k=n}^{\infty} \frac{\alpha_{k}}{k^{2 / p}}, \\
& \alpha_{k}=C\left(k Q_{k}-(k-1) Q_{k-1}\right), \quad k \geq 1, \lim _{n \rightarrow \infty} \frac{\beta_{n}}{n^{1 / p}}=0 .
\end{aligned}
$$

And

$$
\begin{aligned}
& E\left(\max _{1 \leq l \leq n}\left|\frac{S_{l}}{l^{1 / p}}\right|^{2}\right) \leq 4 \sum_{l=1}^{n} \frac{\alpha_{l}}{l^{2 / p}}<\infty, \\
& E\left(\sup _{l \geq 1}\left|\frac{S_{l}}{l^{1 / p}}\right|^{2}\right) \leq 4 \sum_{l=1}^{\infty} \frac{\alpha_{l}}{l^{2 / p}}<\infty .
\end{aligned}
$$


If we further assume that $\alpha_{n}>0$ for infinitely many $n$, then

$$
E\left(\sup _{l \geq 1}\left|\frac{S_{l}}{\beta_{l}}\right|^{2}\right) \leq 4 \sum_{l=1}^{\infty} \frac{\alpha_{l}}{\beta_{l}^{2}}<\infty
$$

In addition, for any $0<r<2$,

$$
E\left(\sup _{l \geq 1}\left|\frac{S_{l}}{l^{1 / p}}\right|^{r}\right) \leq 1+\frac{4 r}{2-r} \sum_{l=1}^{\infty} \frac{\alpha_{l}}{l^{2 / p}}<\infty
$$

Proof Since $\left\{X_{n}, n \geq 1\right\}$ is a sequence of mean zero random variables satisfying the maximal moment inequality with exponent 2 , we have

$$
E\left(\max _{1 \leq j \leq n}\left|\sum_{k=1}^{j} X_{k}\right|^{2}\right) \leq C \sum_{k=1}^{n} E X_{k}^{2} \leq C n Q_{n}=\sum_{k=1}^{n} \alpha_{k} .
$$

And we can obtain $\alpha_{k} \geq 0$ for all $k \geq 1$ from its definition. Denote $b_{n}=n^{1 / p}$ and $\Lambda_{n}=$ $\sum_{k=1}^{n} \alpha_{k}, n \geq 1$. By (3.23), we can get

$$
\sum_{l=1}^{\infty} \Lambda_{l}\left(\frac{1}{b_{l}^{2}}-\frac{1}{b_{l+1}^{2}}\right)=C \sum_{l=1}^{\infty} l Q_{l}\left(\frac{1}{l^{2 / p}}-\frac{1}{(l+1)^{2 / p}}\right) \leq \frac{2 C}{p} \sum_{l=1}^{\infty} \frac{Q_{l}}{l^{2 / p}}<\infty .
$$

Thus (2.4) holds. It follows from Remark 2.1 in [16] that (2.4) implies (2.5). By Lemma 2.5, we can get (3.24)-(3.29) immediately. It follows from (3.28) that

$$
\begin{aligned}
E\left(\sup _{l \geq 1}\left|\frac{S_{l}}{l^{1 / p}}\right|^{r}\right) & =\int_{0}^{\infty} P\left(\sup _{l \geq 1}\left|\frac{S_{l}}{l^{1 / p}}\right|^{r}>t\right) d t \\
& =\int_{0}^{1} P\left(\sup _{l \geq 1}\left|\frac{S_{l}}{l^{1 / p}}\right|^{r}>t\right) d t+\int_{1}^{\infty} P\left(\sup _{l \geq 1}\left|\frac{S_{l}}{l^{1 / p}}\right|^{r}>t\right) d t \\
& \leq 1+E\left(\sup _{l \geq 1}\left|\frac{S_{l}}{l^{1 / p}}\right|^{2}\right) \int_{1}^{\infty} t^{-2 / r} d t \\
& \leq 1+\frac{4 r}{2-r} \sum_{l=1}^{\infty} \frac{\alpha_{l}}{l^{2 / p}}<\infty .
\end{aligned}
$$

The proof is completed.

Remark 3.4 It is easy to see that a wide class of mean zero random variables satisfies the maximal moment inequality with exponent 2 . Examples include independent random variables, negatively associated random variables (see Matula [20]), negatively superadditive dependent random variables (see Shen et al. [12]), $\varphi$-mixing random variables and AANA random variables (see Wang et al. [21, 22]), and $\tilde{\rho}$-mixing random variables (see Utev et al. [23]). So Theorems 3.1-3.3 hold for this wide class of random variables. 
Authors' contributions

All authors read and approved the final manuscript.

\section{Acknowledgements}

The authors are most grateful to the editor and the anonymous referee for their careful reading and insightful comments. This work is supported by the National Natural Science Foundation of China (11171001, 11201001), Natural Science Foundation of Anhui Province (1208085QA03), Humanities and Social Sciences Project from Ministry of Education of China (12YJC91007), Key Program of Research and Development Foundation of Hefei University (13KY05ZD) and Doctoral Research Start-up Funds Projects of Anhui University.

\section{Received: 9 August 2013 Accepted: 4 November 2013 Published: 25 Nov 2013}

\section{References}

1. Adler, A, Rosalsky, A: On the strong law of large numbers for normed weighted sums of i.i.d. random variables. Stoch. Anal. Appl. 5, 467-483 (1987)

2. Chow, YS, Teicher, H: Almost certain summability of independent, identically distributed random variables. Ann Math. Stat. 42, 401-404 (1971)

3. Fernholz, LT, Teicher, H: Stability of random variables and iterated logarithm laws of martingales and quadratic forms. Ann. Probab. 8, 765-774 (1980)

4. Jamison, B, Orey, S, Pruitt, W: Convergence of weighted averages of independent random variables. Z. Wahrscheinlichkeitstheor. Verw. Geb. 4, 40-44 (1965)

5. Teicher, H: Almost certain convergence in double arrays. Z. Wahrscheinlichkeitstheor. Verw. Geb. 69, 331-345 (1985)

6. Rosalsky, A: Strong stability of normed weighted sums of pairwise i.i.d. random variables. Bull. Inst. Math. Acad. Sin. 15, 203-219 (1987)

7. Sung, SH: Strong law of large numbers for weighted sums of pairwise independent random variables. Bull. Inst. Math. Acad. Sin. 27(1), 23-28 (1999)

8. Sung, $\mathrm{SH}$ : On the strong law of large numbers for pairwise i.i.d. random variables with general moment conditions. Stat. Probab. Lett. 83, 1963-1968 (2013)

9. Wu, QY: A strong limit theorem for weighted sums of sequences of negatively dependent random variables. J. Inequal. Appl. 2010, Article ID 383805 (2010)

10. $\mathrm{Wu}, \mathrm{QY}$, Jiang, YY: Some strong limit theorems for weighted product sums of $\tilde{\varphi}$-mixing sequences of random variables. J. Inequal. Appl. 2009, Article ID 174768 (2009)

11. Hu, SH, Wang, XJ, Yang, WZ, Zhao, T: The Hàjek-Rènyi-type inequality for associated random variables. Stat. Probab. Lett. 79, 884-888 (2009)

12. Shen, Y, Wang, XJ, Yang, WZ, Hu, SH: Almost sure convergence theorem and strong stability for weighted sums of NSD random variables. Acta Math. Sin. Engl. Ser. 29, 743-756 (2013)

13. Zhou, XC, Tan, CC, Lin, JG: On the strong laws for weighted sums of $\rho^{*}$-mixing random variables. J. Inequal. Appl. 2011, Article ID 157816 (2011)

14. Zhou, XC: Complete moment convergence of moving average processes under $\varphi$-mixing assumptions. Stat. Probab. Lett. 80, 285-292 (2010)

15. Sung, $\mathrm{SH}$ : On the strong law of large numbers for weighted sums of random variables. Comput. Math. Appl. 62, 4277-4287 (2011)

16. Hu, SH: Some new results for the strong law of large numbers. Acta Math. Sin. (Chin. Ser.) 46(6), 1123-1134 (2003) (Chinese)

17. Fazekas, I, Klesov, O: A general approach to the strong law of large numbers. Theory Probab. Appl. 45(3), 436-449 (2002)

18. Rao, MM: Measure Theory and Integration. Wiley, New York (1987)

19. Hu, SH, Hu, M: A general approach rate to the strong law of large numbers. Stat. Probab. Lett. 76, $843-851$ (2006)

20. Matula, P: A note on the almost sure convergence of sums of negatively dependent random variables. Stat. Probab. Lett. 12, 209-213 (1992)

21. Wang, XJ, Hu, SH, Shen, Y, Yang, WZ: Moment inequality for $\varphi$-mixing sequences and its applications. J. Inequal. Appl. 2009, Article ID 379743 (2011)

22. Wang, XJ, Hu, SH, Yang, WZ: Convergence properties for asymptotically almost negatively associated sequence. Discrete Dyn. Nat. Soc. 2010, Article ID 218380 (2010)

23. Utev, S, Peligrad, M: Maximal inequalities and an invariance principle for a class of weakly dependent random variables. J. Theor. Probab. 16, 101-115 (2003)

10.1186/1029-242X-2013-563

Cite this article as: Shen et al.: On strong law of large numbers and growth rate for a class of random variables. Journal of Inequalities and Applications 2013, 2013:563 